
\title{
25 Research Soure \\ Comparison of CISNE and MASCC Score in Predicting Complications on Post Chemotherapy Febrile Neutropenia
}

Sharifah Shakinah

Universitas Indonesia https://orcid.org/0000-0001-6513-282X

Erni Nelwan ( $\nabla$ ejnelwan@yahoo.com )

University of Indonesia / Cipto Mangunkusumo General Hospital https://orcid.org/0000-0003-40645412

Anna Lubis

Universitas Indonesia Fakultas Kedokteran

Robert Sinto

Universitas Indonesia Fakultas Kedokteran

Herdiman Pohan

Universitas Indonesia Fakultas Kedokteran

\section{Research note}

Keywords: post-chemotherapy febrile neutropenia, CISNE score, MASCC score, complications, solid malignancy, hematologic malignancy

Posted Date: March 19th, 2021

DOI: https://doi.org/10.21203/rs.3.rs-322271/v1

License: (c) (1) This work is licensed under a Creative Commons Attribution 4.0 International License. Read Full License 


\section{Abstract}

Objective Despite the routine use of MASCC score in febrile neutropenia patients, researches showed that serious complications still occur in $15-42 \%$ of low-risk patients; therefore, a new scoring system Clinical Index of Stable Febrile Neutropenia (CISNE) is developed to predict the complication better. This retrospective cohort study aimed to assess the role of CISNE score compared to MASCC score as a risk stratification in post-chemotherapy FN in solid and hematologic malignancy. Patients who underwent inpatient treatment with FN between July 2015 and December 2019 were grouped based on both scores, while complications during inpatient was recorded. Both score's areas under the receiver operating characteristics curve were compared using DeLong method.

Results CISNE score showed a better performance both in solid malignancy with AUC of CISNE score $(0.893 ; 95 \% \mathrm{Cl} 0.829-0.95, \mathrm{p}=0.03)$ compared to AUC of MASCC score $(0.77 ; 95 \% \mathrm{Cl} 0.68-0,86, \mathrm{p}=$ $0.04)$ and in hematologic malignancy with AUC of CISNE score $(0.91 ; 95 \% \mathrm{Cl} 0.84-0.97, \mathrm{p}=0.03)$ and AUC MASCC score $(0.735 ; 95 \% \mathrm{Cl} 0.68-0.86, \mathrm{p}=0.04)$. CISNE score showed a better diagnostic performance with cut-off point value of 2 .

\section{Introduction}

Febrile neutropenia (FN) is an oncologic emergency that occurs in 20 cases per 1000 patients who undergo chemotherapy. FN is a life-threatening condition with a $12.5 \%$ mortality rate.[1, 2] Risk stratification in FN plays an important role in increasing the accuracy of therapy, thus reducing complications. The Infectious Diseases Society of America (IDSA) recommends using Multinational Association for Supportive Care in Cancer (MASCC) scores to differentiate between low-risk and high-risk patients and select the appropriate antibiotics.[3-5] Despite the routine use of MASCC scores in daily practice, researches have shown that severe complications still occur in $15-42 \%$ of low-risk patients.[3, 4], [6] Therefore, a new scoring system, the Clinical Index of Stable Febrile Neutropenia (CISNE), was developed to predict such complications better. However, several studies have been conducted to assess the performances of CISNE scores, and the results remain controversial.[2, 6-10] Further study comparing CISNE score with MASCC score based on tumor type is still needed to assess the role of each score in predicting complications and therefore reducing mortality.

\section{Methods}

This is a retrospective cohort study to assess the performances of CISNE and MASCC scores in predicting complications in FN patients with solid and hematological malignancy. We collected data from medical records of patients with chemotherapy-induced FN underwent inpatient treatment in Cipto Mangunkusumo General Hospital from January 2015 to December 2019. Inclusion criteria included the following: above 18 years of age, fulfilling the diagnostic criteria of $\mathrm{FN}$, and receiving appropriate antibiotics based on IDSA guideline. The exclusion criteria were acute leukemia, incomplete medical records, and FN unrelated to chemotherapy. Data was collected through consecutive sampling and 
processed with STATA software. Baseline characteristics were presented in numerical and categorical data with percentages and medians with interquartile range. CISNE and MASCC scores were analyzed using the area under the receiver operating characteristic (AUROC). We used the Liu method to determine the cut-off point and the DeLong method in comparing the AUROC between the CISNE and MASCC scores. Diagnostic study of each score was calculated based on the $2 \times 2$ table. Our research was approved by the ethics committee of Faculty of Medicine, University of Indonesia.

\section{Results}

We found 95 cases of FN that had resulted from chemotherapy treatment of a solid malignancy and 73 cases of FN that had resulted from chemotherapy treatment of a hematologic malignancy. Febrile neutropenia was found in $51.8 \%$ of men under 60 years old. In the solid malignancy group, we found $46.32 \%$ cases of FN that had resulted from chemotherapy treatment of a head and neck tumor. We found $87.67 \%$ cases of FN in patients with non-Hodgkin's lymphoma who underwent chemotherapy in the hematologic malignancy group. Febrile neutropenia was found in $39.88 \%$ of patients after the first cycle of chemotherapy. We found that $55.36 \%$ of FN patients had been admitted with a secondary infection, and $51.5 \%$ of the group were diagnosed with pneumonia. (Table 1) Complications during admission were found in $45.83 \%$ of patients, $83.1 \%$ of which were diagnosed as septicemia. 


\begin{tabular}{|c|c|}
\hline Characteristic & Number (\%) \\
\hline \multicolumn{2}{|l|}{ Gender } \\
\hline - Women, n (\%) & $81(48,2)$ \\
\hline - Men, n (\%) & $87(51,8)$ \\
\hline Age, median, (IQR) & $49(36,5-59,5)$ \\
\hline \multicolumn{2}{|l|}{ Age group } \\
\hline$-<60$ year old, $n(\%)$ & $126(75)$ \\
\hline - $\geq 60$ year old, $n(\%)$ & $42(25)$ \\
\hline \multicolumn{2}{|l|}{ Solid tumor $(n=95)$} \\
\hline - Head and neck, n (\%) & $44(46,32)$ \\
\hline - Lung, n (\%) & $2(2,11)$ \\
\hline - Breast, n (\%) & $12(12,63)$ \\
\hline - Gastrointestinal, n (\%) & $4(4,21)$ \\
\hline - Genitourinary, n (\%) & $6(6,32)$ \\
\hline - Bone and soft tissue, n (\%) & $17(17,89)$ \\
\hline - Gynecology, n (\%) & $9(9,47)$ \\
\hline - Thymic carcinoma, n (\%) & $1(1,05)$ \\
\hline \multicolumn{2}{|l|}{ Hematologic malignancy $(n=73)$} \\
\hline - Limfoma Non Hodgkin, n (\%) & $64(87,67)$ \\
\hline - Limfoma Hodgkin, n (\%) & $3(4,11)$ \\
\hline - Myeloma, n (\%) & $4(5,48)$ \\
\hline - Chronic leukemia, n (\%) & $2(2,74)$ \\
\hline Onset of fever (IQR) & $7(4-10)$ \\
\hline \multicolumn{2}{|l|}{ Aim of chemotherapy } \\
\hline - Definitive, n (\%) & $84(50)$ \\
\hline - Paliative, n (\%) & $84(50)$ \\
\hline
\end{tabular}




\begin{tabular}{|ll|}
\hline Characteristic & Number (\%) \\
\hline Staging & \\
- Stage 1, n (\%) & $3(1,7)$ \\
- Stage 2, n (\%) & $23(13,6)$ \\
- Stage 3, n (\%) & $21(12,5)$ \\
- Stage 4, n (\%) & $121(72,2)$ \\
\hline Chemotherapy cycle & \\
- 1, n (\%) & $67(39,88)$ \\
- 2, n (\%) & $26(15,48)$ \\
- 3, n (\%) & $20(11,9)$ \\
- 4, n (\%) & $24(14,29)$ \\
- 5, n (\%) & $11(6,55)$ \\
- 6, n (\%) & $20(11,9)$ \\
\hline Chemotherapy intensity based on NCCN & \\
- Low risk & $3(1,79)$ \\
- Moderate risk & $80(47,62)$ \\
- High risk & $85(50,6)$ \\
\hline Documented infection & \\
- Documented infection, n (\%) & $93(9,7)$ \\
- Without documented infection, n (\%) & $75(44,64)$ \\
\hline Infection & $5(5,3)$ \\
- Pneumonia, n (\%) & $21(22,5)$ \\
- Urinary tract infection, n (\%) & $91,5)$ \\
- Gastrointestinal infection, n (\%) & \\
- Skin and soft tissue infection, n (\%) & \\
- Oral, ear, nose, throat infection (\%) & \\
\hline
\end{tabular}

The CISNE scores effectively predicted febrile neutropenia complications resulting from chemotherapy treatment of a solid malignancy with an AUROC of $0.893(\mathrm{Cl} 95 \% 0.829-0.95 ; \mathrm{p}=0.03)$, whereas the MASCC scores with an AUROC of $0.77(\mathrm{Cl} 95 \% 0.68-0.86 ; p=0,04)$. Therefore, CISNE scores were found 
to be significantly better at predicting complications than MASCC scores in solid malignancy. CISNE scores also effectively predict complications in febrile neutropenia resulting from chemotherapy in hematologic malignancies with an AUROC of 0.91 ( $\mathrm{Cl} 95 \%$ 0.84-0.97; $p=0,03$, whereas MASCC scores with an AUROC of $0.835(\mathrm{Cl} 95 \% 0.68-0.86 ; p=0,04)$. We also found a marked difference in our work than in previous research. In patients with solid and hematologic malignancies, we found that CISNE score has a better performance with cut-off point of 2 and MASCC score has a better performance with a cut-off point of 20 .

\section{Discussion}

This study was the first study to assess CISNE and MASCC score performance based on malignancy type. Previous studies by Koppaka et al.[11] and Moon et al.[7] were only conducted on patients with solid malignancy. Other research by Bayonas et al.[2] was conducted on patients with solid malignancy in $90 \%$ of cases. The CISNE score itself was originally developed by Bayonas et al.[2, 12] in a solid malignancy population, and thus its performance in hematologic malignancy is unknown. The incidence of neutropenia in hematologic and solid malignancy display different characteristics; for instance, neutropenia periods are longer, and positive culture results are more often found in patients with hematologic malignancy.[13] In our study, there were no significant differences in the characteristics of patients with solid tumors and hematologic malignancy subjects. The efficacies of CISNE scores and MASCC scores were assessed based on each malignancy type.

Furthermore, the types of solid tumor in this study differed from the types of solid tumor in previous studies. In a study by Bayonas et al.[2] and Moon et al.,[7] the majority of solid tumor were breast cancer. In this study, the majority of FN occurrences in solid tumors were head and neck tumors (46.32\%), followed by bone and soft tissue tumor (17.89\%). These findings showed a difference in the prevalence of tumors in our study location, because as the national referral hospital we received many end-stage cancer patients with different types of tumor distribution.[14, 15]

In hematologic malignancy subjects, $87.67 \%$ of patients had non-Hodgkin's lymphoma. We excluded patients with acute leukemia because febrile neutropenia in acute leukemia patients, according to the IDSA guidelines, are in the high-risk category, regardless of their MASCC scores. Aplasia in patients with acute leukemia is influenced by many factors, including those associated with the disease (e.g., leukocyte function defects, or humoral immunity deficiencies); patient-dependent factors (e.g., age, comorbidities, or malnutrition); or the effects of chemotherapy (e.g., prolonged aplasia due to high-intensity chemotherapy, bacterial colonization due to aplasia, recurrent antibiotic use, or central catheter-related infection) therefore we exclude acute leukemia in our study.[16]

By the time of FN diagnosis, 55.36\% subjects had documented infections, a greater number than in previous studies, which recorded an infection rate around 30\% in FN patients.[17] In subjects with documented infection, $51.5 \%$ had pneumonia, a common comorbidity in neutropenic patients. Vehreschild et al.[18] showed that $36.4 \%$ of the neutropenic patients in their study had pneumonia. In 
addition to pneumonia, some subjects also displayed gastrointestinal infection in the form of acute gastroenteritis and diarrhea. These conditions in neutropenic patients are also known as neutropenic enterocolitis, which displays an incidence estimated around $5.6 \%$. Such infections can occur as a direct result of taxane-class chemotherapy, which can damage the mucosal surface.[19]

The median of the absolute neutrophil count (ANC) was 212 cells, which categorized in severe neutropenia. The risk of infection and complications in patients with FN becomes higher as ANC values drop. $[20,21]$ Procalcitonin levels were examined in $64.2 \%$ of subjects, with a median value of $2.66 \mathrm{ng} / \mathrm{ml}$. Procalcitonin is a significant parameter to distinguish infections as a cause of FN. A systematic study by Sakr et al.[22] obtained a cut-off value of $0.5-2 \mathrm{ng} / \mathrm{ml}$ for indicating life-threatening conditions in neutropenic patients. Increased procalcitonin levels were more pronounced in patients with bacteremia or sepsis, and the mean value of $2.66 \mathrm{ng} / \mathrm{mml}$ illustrated a serious condition encountered in our subjects.

Complications were found in $45.83 \%$ of subjects, a greater prevalence than previous studies. For example, in an experiment by Ahn et al and Coyne eatl., 8 , [9] complications occurred in $20.8 \%$ and $25.7 \%$ of subjects, respectively. This difference might occur because of the different settings from which subjects were recruited. Coyne et al. and Ahn et al. recruit subjects from the emergency wing triage so that clinically stable patients were included in the subject. In contrast, in this study, we did not include outpatient data. This was also illustrated in the percentage of subjects classified in the low-risk category by MASCC and CISNE scores. In this study, based on MASCC scores, $58.3 \%$ of participants were included in the low-risk category. These results differed from those found by Ahn et al and Coyne et al., in which $89.1 \%$ and $73.5 \%$ of subjects were at low risk, respectively. The population in this study showed a higher prevalence of complications. In those who appeared to have a complication, $83.1 \%$ were diagnosed with sepsis, and $72.7 \%$ underwent shock. This is higher than the previous study that estimated the prevalence of this sepsis in $\mathrm{FN}$ patient ranges from 7 to $45 \%$.[21]

This study analyzes the performance of CISNE scores compared with MASCC scores in predicting complications in FN patients with solid and hematologic malignancies after chemotherapy. In patients with solid malignancies, CISNE scores performed well, with an AUROC of 0.89. Two previous studies have analyzed the performance of CISNE scores in patients with solid malignancies. Research by Koppaka et al.[11] also showed that CISNE scores had an AUROC of 0.846, whereas in Moon et al.[7], CISNE scores had an AUROC of only 0.66 . In our study, the population of solid malignancies was dominated by headneck malignancies, whereas previous studies showed head-neck malignancies in only $5 \%$ of populations. Also, these research subjects were recruited in different settings. Previous studies gathered subjects from emergency wings and outpatient, whereas in our study, subjects were recruited from hospitalized FN patients. The CISNE score in the study by Bayonas et al.[2] was divided into three groups: low, moderate, and high risk, with a sensitivity of $80.1 \%$ and a specificity of $75.6 \%$. Koppaka et al. used cut-off point 2 to divide patients into low risk and high risk groups with a sensitivity of $80.6 \%$ and specificity of $72.8 \%$, a positive predictive value of $35.2 \%$, and a negative predictive value of $95.3 \%$. We conducted an analysis to assess the cut-off point and obtained a sensitivity of $74.5 \%$, a specificity of 
$86.4 \%$, a positive predictive value of $86.4 \%$, and a negative predictive value of $74.5 \%$, and cut-off point of 2.

This is the first study to analyze the effectiveness of CISNE scores in predicting complications of FN after chemotherapy in patients with hematologic malignancy. The analysis showed that the CISNE scores perform well, with an AUROC of 0.91. Previous research by Mohindra et al.[10] was not specific to hematologic malignancy, but this group also conducted a subgroup analysis of the hematologic malignancy population and obtained an AUROC of 0.67 . We performed the cut-off analysis to get the best diagnostic value. We obtained a cut-off point of 2 with a sensitivity of $78.9 \%$, a specificity of $91.4 \%$, a positive predictive value of $90.9 \%$, and a negative predictive value of $80 \%$. There was a significant difference between the performances of the CISNE and MASCC scores in predicting complications in patients with FN that had resulted from hematologic malignancy chemotherapy. These results showed that CISNE scores effectively predict complications in febrile neutropenia patients, both in those with solid and hematologic malignancies. These differences between CISNE and MASCC scores may result from different score components. CISNE scores assess patient conditions with ECOG performance, whereas MASCC scores assess patients' burden of disease. The use of ECOG performance can give a more objective assessment than the burden of disease, which is highly dependent on clinician assessment. These objective assessments resulted in less misclassification of patients.[6]

Our study showed that the use of CISNE scores to substitute MASCC scores in risk classification of FN patients, both solid and hematologic malignancies, can reduce misclassification. Further research on the role of CISNE scores in the selection of antibiotic therapy may be needed. We found that re-classification of CISNE scores into two groups (low risk and high risk) with a cut-off point of 2 was more effective in predicting complications, thus helping clinicians determine patient therapy.

\section{Limitations}

Our study limitation was a retrospective study using secondary data obtained from medical records; therefore some subjective score components did not have standard parameters. This source of medical record was also the cause why the demographic data of the type of tumor is different from the national or regional characteristics

\section{Abbreviations}

ANC: Absolute Neutrophil Count

AUROC: Area Under Receiver Operating Characteristic

CISNE: Clinical Index of Stable Febrile Neutropenia

FN: Febrile Neutropenia

IDSA: Infectious Diseases Society of America 


\section{Declarations}

\section{Ethics approval and consent to participate}

This study was approved by Ethics Committee of the Faculty of Medicine, the Univerisity of Indonesia which complied with Declaration of Helsinki. No intervention in this study influenced the outcome of subjects. Privacy and confidentiality were taken into consideration throughout the research periode by not sharing any information collected from the study.

\section{Availability of data and material}

Data available from the corresponding author on rational request.

\section{Funding}

No grant or funding supports have been received for this study

\section{Acknowledgements}

We are grateful to all of study participants and everyone helped for this study to run well.

\section{Consent for publication}

Not applicable

\section{Competing interest}

The authors declare that they have no competing interests.

\section{Authors contributions}

SS proposed the topic, SS designed and collect data under direction of EJN, AML, RS, and HTP. All the results were discussed by SS together with EJN, AML, RS, and HTP. The revision was mainly done by SS; EJN, AML, RS provided some recommendations for the revision. THP gave final comments to the manuscript before being resubmitted. All authors read and approved the final manuscript. 


\section{References}

1. Schelenz S, Giles D, Abdallah S. Epidemiology, management and economic impact of febrile neutropenia in oncology patients receiving routine care at a regional UK cancer centre. Ann Oncol. 2012;23:1889-93.

2. Carmona-Bayonas A, Jiménez-Fonseca P, Echaburu JV, Antonio M, Font C, Biosca M, et al. Prediction of Serious Complications in Patients With Seemingly Stable Febrile Neutropenia: Validation of the Clinical Index of Stable Febrile Neutropenia in a Prospective Cohort of Patients From the FINITE Study. Journal of Clinical Oncology. 2015;33:465-70.

3. Klastersky J, Paesmans M, Rubenstein EB, Boyer M, Elting L, Feld R, et al. The Multinational Association for Supportive Care in Cancer Risk Index: A Multinational Scoring System for Identifying Low-Risk Febrile Neutropenic Cancer Patients. JCO. 2000;18:3038-51.

4. Klastersky, J N J, Rolston, K, Rapoport, B, Maschmeyer, G, Appro, M, Herrstedt, J. Management of Febrile Neutropaenia: ESMO Clinical Practice Guidelines | ESMO. Ann Oncol. 27:111-8.

5. Freifeld AG, Bow EJ, Sepkowitz KA, Boeckh MJ, Ito JI, Mullen CA, et al. Clinical Practice Guideline for the Use of Antimicrobial Agents in Neutropenic Patients with Cancer: 2010 Update by the Infectious Diseases Society of America. Clin Infect Dis. 2011;52:e56-93.

6. Bitar RA. Utility of the Multinational Association for Supportive Care in Cancer (MASCC) Risk Index Score as a Criterion for Nonadmission in Febrile Neutropenic Patients with Solid Tumors. Perm J. 2015;19:37-47.

7. Moon H, Choi YJ, Sim SH. Validation of the Clinical Index of Stable Febrile Neutropenia (CISNE) model in febrile neutropenia patients visiting the emergency department. Can it guide emergency physicians to a reasonable decision on outpatient vs. inpatient treatment? PLoS ONE. 2018;13:1-12.

8. Coyne CJ, Le V, Brennan JJ, Castillo EM, Shatsky RA, Ferran K, et al. Application of the MASCC and CISNE Risk-Stratification Scores to Identify Low-Risk Febrile Neutropenic Patients in the Emergency Department. Ann Emerg Med. 2017;69:755-64.

9. Ahn S, Rice TW, Yeung S-CJ, Cooksley T. Comparison of the MASCC and CISNE scores for identifying low-risk neutropenic fever patients: analysis of data from three emergency departments of cancer centers in three continents. Support Care Cancer. 2018;26:1465-70.

10. Mohindra R, Mathew R, Yadav S, Aggarwal P. CISNE versus MASCC: Identifying low risk febrile neutropenic patients. The American Journal of Emergency Medicine. 2019.

11. Koppaka D, Kuntegowdanahalli LC, Lokanath D, Babu KG, Jacob LA, Babu MCS, et al. Assessment and comparison of CISNE model versus MASCC model in clinically stable febrile neutropenia patients. Annals of Oncology. 2018;29:ix129.

12. Carmona-Bayonas A, Jiménez-Fonseca P, Virizuela J, Antonio M, Font $C$, Biosca $M$, et al. Performance of the clinical index of stable febrile neutropenia (CISNE) in different types of infections and tumors. Clin TransI Oncol. 2017;19:386-95. 
13. Al-Ahwal MS, Johar I, Al-Sayws, Fatih. Febrile Neutropenia Comparison Between Solid Tumours and Hematological Malignancies. Pan Arab Medical Journal. 2005;2:4-7.

14. Hassan BAR, Yusoff ZBM, Othman SB. A Close Look at Neutropenia among Cancer Patients - Risk Factor and Management. Updates on Cancer Treatment. 2015.

15. Budiana ING, Febiani M. Febrile Neutropenia pada Pasien Pascakemoterapi. Indonesian Journal of Cancer. 2017;11:77-82.

16. Hansen B-A, Wendelbo $\emptyset$, Bruserud $\emptyset$, Hemsing AL, Mosevoll KA, Reikvam H. Febrile Neutropenia in Acute Leukemia. Epidemiology, Etiology, Pathophysiology and Treatment. Mediterr J Hematol Infect Dis. 2019;12.

17. Punnapuzha S, Edemobi PK, Elmoheen A. Febrile Neutropenia. In: StatPearls. Treasure Island (FL): StatPearls Publishing; 2019. http://www.ncbi.nlm.nih.gov/books/NBK541102/. Accessed 3 Dec 2019.

18. Vehreschild JJ. Pneumonia and Lung Infiltrates in Neutropenic Patients: Many Stones Unturned. Annals ATS. 2013;10:493-5.

19. Rodrigues FG, Dasilva G, Wexner SD. Neutropenic enterocolitis. World J Gastroenterol. 2017;23:42-7.

20. Cancer NCC for. Diagnosis of neutropenic sepsis. National Institute for Health and Clinical Excellence (UK); 2012. https://www.ncbi.nlm.nih.gov/books/NBK373673/. Accessed 1 Jul 2020.

21. Kochanek M, Schalk E, von Bergwelt-Baildon M, Beutel G, Buchheidt D, Hentrich M, et al. Management of sepsis in neutropenic cancer patients: 2018 guidelines from the Infectious Diseases Working Party (AGIHO) and Intensive Care Working Party (iCHOP) of the German Society of Hematology and Medical Oncology (DGHO). Ann Hematol. 2019;98:1051-69.

22. Sakr Y, Sponholz C, Tuche F, Brunkhorst F, Reinhart K. The role of procalcitonin in febrile neutropenic patients: review of the literature. Infection. 2008;36:396-407.

\section{Figures}




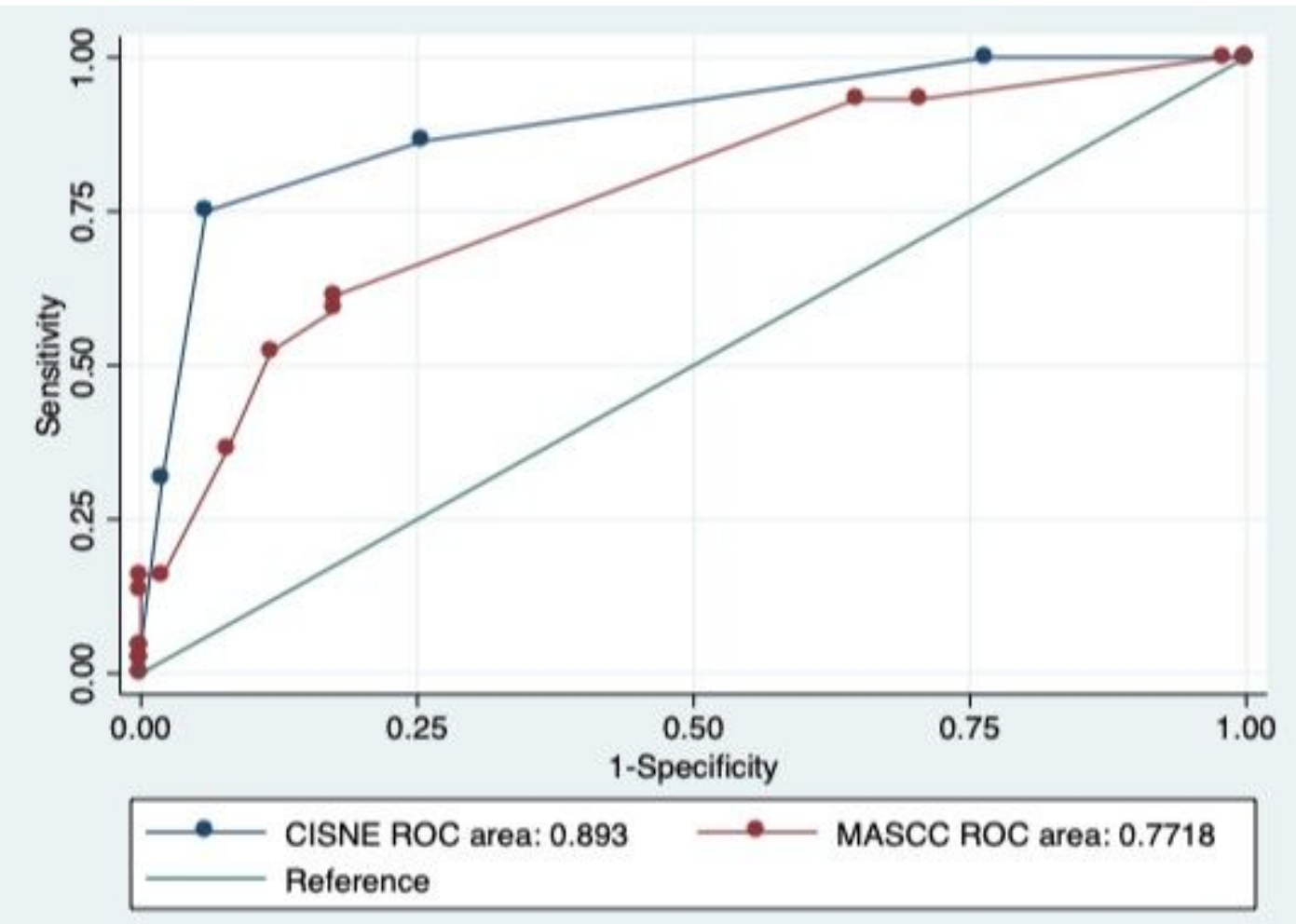

Figure 1

AUROC comparation of CISNE and MASCC score in solid tumor

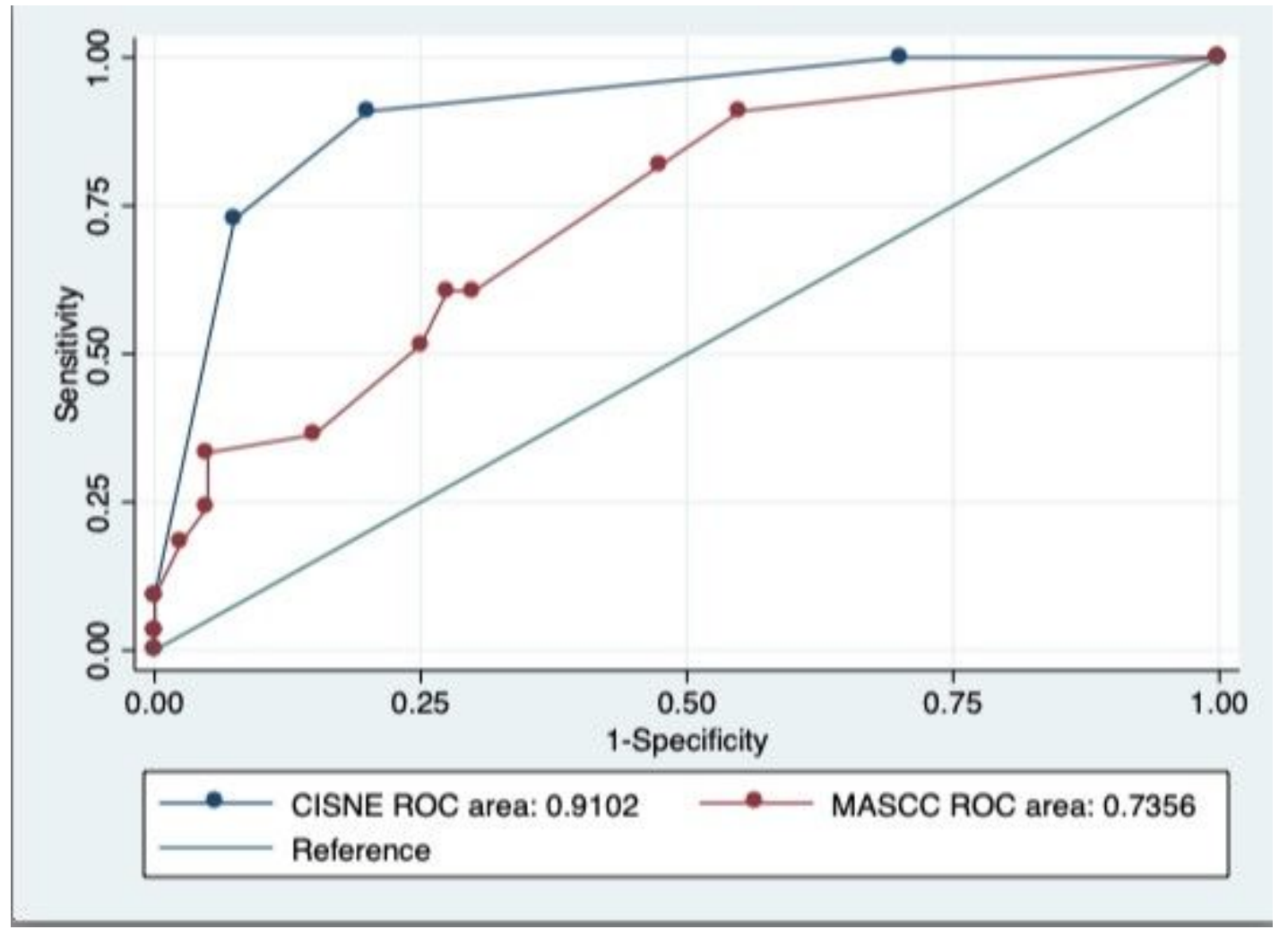

Figure 2

AUROC comparation of CISNE and MASCC score in hematologic malignancy 


\section{Supplementary Files}

This is a list of supplementary files associated with this preprint. Click to download.

- SupplementaryMaterial.docx 